\title{
On the Individual Expectations of Non-Average Investors
}

\author{
Lucia Del Chicca, Gerhard Larcher \\ Institute of Financial Mathematics, University of Linz, Altenberger Strasse Linz, Austria \\ E-mail:\{lucia.delchicca, gerhard.larcher\}@jku.at \\ Received August 25, 2011; revised October 1, 2011; accepted October 15, 2011
}

\begin{abstract}
An "average investor" is an investor who has "average risk aversion", "average expectations" on the market returns and should invest in the "market portfolio" (this is, according to the Capital Asset Pricing Model, the best possible portfolio for such an investor). He is compared with a "non-average investor". This - in our setting - is an investor who has the same "average risk aversion" but invests in other investment strategies, for example options. Such a "non-average investor" must consequently have expectations on the market return that are different from the average: the "non-average expectations". In this paper we give an explicit formula for the "non-average expectations" in an arbitrary $N$-step model and for the extended concept in a BlackScholes model, in the path-independent case and in the path-dependent case. Further we explicitly classify all the investment strategies for which the resulting "non-average expectations" show this mean aversion property. Various examples are given in the paper. These investigations were part of more general investigations initiated by an investment company carrying out certain subtle option trading strategies.
\end{abstract}

Keywords: Binomial Model, Black-Scholes Model, Options, Expectations, Mean Averting Strategies

\section{Introduction}

Often it is interesting for fonds-managers, asset managers, or consultants to know which kind of investor is appropriate to a certain strategy. So in this work we give an answer to the following question: "which sort of investor (differing from the average) is interested in trading a given (alternative) strategy?" This question occurs amongst others in the field of behavioral finance. It deals with the psychology of investors and the consequences of their expectations about the market which lead to investment decisions. We give an answer to this question with the help of certain mathematical model first introduced by $\mathrm{H}$. Leland.

In two inspiring articles [1,2] Leland trys to identify the characteristics of investors who buy or sell European call options or other path-independent or path-dependent contingent claims. In both papers Leland considers investors who trade in those options just out of speculative reasons. In the first article he concentrates on investors who have the same return expectations as an average investor and he asks for their individual risk aversion. In the second article he considers investors with the same risk aversion as the average investor and he studies their differing expectations on the market return.

Our work will be based on this second article. In this article Leland considers an asset (market portfolio) in a binomial 3-step model and an "average investor" with given "average expectations" on the market returns, and with "average risk aversion", i.e. with a utility function $U$ for which the above market portfolio maximizes the utility of the average investor. This "average investor" is compared with a "non-average investor" who has the same utility function $U$, but who follows investment strategies differing from just buying the market portfolio. Especially in [2] certain basic types of (path-dependent and of path-independent) option strategies in the binomial 3-step model are considered. As Leland points out the average investor will never purchase (or sell) fairly-priced options since options are in zero net supply. Thus investors holding options must differ from average, i.e., in our setting, their expectations on the market return must differ from the "average expectations". For more-step models Leland asserts that this mean-aversion can be found especially at nodes with stock-value close to the initial stock-value. For path-dependent (e.g. Asian or lookback call) contingent claim traders Leland detects "somewhat diffuse" return expectations.

It is the aim of this paper to fully discuss the above modelling in a general binomial $N$-step model and subsequent in a Black-Scholes model, and to give a complete answer to the following questions: 
1) Can we give an explicit formula for the "non-average expectations" in an arbitrary $N$-step model and extend the concept in a Black-Scholes model, as well in the pathindependent case as in the path-dependent case?

2) Can we explicitly classify all the investment strategies for which the resulting "non-average expectations" show this mean-aversion property?

3) To what extent do the conclusions of Leland hold for $\mathrm{N}$-step models, the Black-Scholes model and for arbitrary trading strategies?

The paper is organized as follows:

In Section 2 we repeat Leland's setting and give all necessary definitions. Especially, we give an exact definition of a "strictly mean-averting trader".

In Section 3 and in Section 4 we discuss the binomial 2-step model for path-independent contingent claims, respectively for path-dependent contingent claims in full detail. (Later, the general cases can be reduced to the 2-step case to some extent.)

In Section 5 we provide the explicit computation technique for the expectations of traders of path-independent as well as path-dependent contingent claims in the $N$-step model.

In Section 6 we show that these expectations imply a certain martingale property.

In Section 7 we explicitly characterize strictly meanaverting respectively mean-reverting investors in pathindependent contingent claims in a binomial $\mathrm{N}$-step model.

In Section 8 we do the same in the continuous case (Black-Scholes model).

In Section 9 we consider as concrete example of (pathindependent) contingent claims, the case of call options.

Finally in Section 10 we consider a special example of path-dependent contingent claims.

Section 11 is devoted to conclusions and a final summary.

\section{Lelands Approach. The Model}

Leland [2] considers an average investor who has average expectations on market returns, average risk aversion (i.e., a common average utility function) and therefore invests in a market portfolio $S$. He assumes that $S$ follows a binomial model. In our paper we will essentially also work in a binomial model as well. Later on, however, we will also consider the Black-Scholes model.

The parameters of the binomial model are given by

$N$ : the number of steps ;

$S_{0}$ : the initial value of the market portfolio;

$u$ : the multiplicator for an up-move;

$d$ : the multiplicator for a down-move, given by $d=1 / u$;

$r$ : the risk free interest rate in the model, assumed to be 0

These parameters determine the risk-neutral probabilities of the model

$$
P_{u}=\frac{e^{r}-\mathrm{d}}{u-\mathrm{d}}=\frac{1}{u+1}
$$

and

$$
P_{d}=\frac{u}{u+1} .
$$

These parameters are fixed for all the investors.

$\pi_{u}$ : is the probability of an up-move from the view of an average investor (the consensus probability) and is timeconstant.

$\pi_{d}$ : the consensus probability of a down move $\pi_{d}=1-\pi_{u}$.

The risk aversion for the average investor is determined by the utility function $U$. We assume that this average investor shares the market expectations of the model for $S$, that he invests in $S$, and that he is a rational investor. So the above market portfolio $S$ maximizes the utility of the average investor. From this assumption we can determine $U$ as it is done in [2] (see also [3]) and we obtain:

with

$$
\begin{gathered}
U(x)=\frac{x^{1-\alpha}}{1-\alpha} \\
\alpha=\frac{\log \left(u \frac{\pi_{u}}{\pi_{d}}\right)}{2 \log u} .
\end{gathered}
$$

Following Leland in [2], we are now interested in investtors with the same risk aversion, i.e. with the same utility function $U$, who, nevertheless, follow other trading strategies than the average investor. Since, by assumption, these investors also maximize their utility, they must have different expectations, i.e. different probabilities for up and down moves in the model. These individual probabilities will be in the center of our interest.

At every node $u^{i} d^{k-i} S_{0} ; i=0, \cdots, k ; k=0, \cdots, N-1$ we will have one but sometimes even several such individual probabilities for an up-move in the next time-step. This depends on whether the individual trading strategy is path-independent or path-dependent. In the latter case at every such node we have $\left(\begin{array}{l}k \\ i\end{array}\right)$ such individual probabilities. For each path

$$
\tilde{S}:=\left(v_{0}, v_{1}, \cdots, v_{k-1}\right)
$$

leading to $u^{i} d^{k-i} S_{0}$ we have exactly one probability

$$
p(\tilde{S} ; k)=p\left(\left(v_{0}, v_{1}, \cdots, v_{k-1}\right) S_{0} ; k\right) .
$$

Here $v_{i} \in\{u, d\}$ stands for a $v_{i}$-move in step $i+1$. In the path-independent case we just use the notation $p\left(u^{i} d^{k-i} S_{0} ; k\right)$. Sometimes, if no confusion is possible, we use notations with reduced information for these individual probabilities for the sake of simplicity. It is the aim to com- 
pute these individual expectations from the individual trading strategies. Note that we carry out a certain "reverse engineering": in usual portfolio theory, once a choice of risk and investor measure are chosen, the optimal portfolio is derived. Here we assume an optimal portfolio under a specific risk measure, and we determine the investor expectations.

In [3] Leland for example considers the following concrete example set of parameters

$$
S_{0}=100 ; \quad u=1.2 ; \quad \pi_{u}=\frac{2}{3},
$$

and an European call-option buyer who is long 1.5 options with strike $K=100$ and who holds an amount of 79.60 in cash. This particular choice is made because of certain norming reasons. The initial value of this portfolio is 100 like the initial value of the market portfolio. We illustrate the situation and Leland's results in Figures 1 and 2.

The probabilities (path-independent) are the computed individual probabilities for up-moves

e.g. $p\left((u, d) S_{0} ; 2\right)=p\left((d, u) S_{0} ; 2\right)=p\left(u d S_{0} ; 2\right)=0.643$.

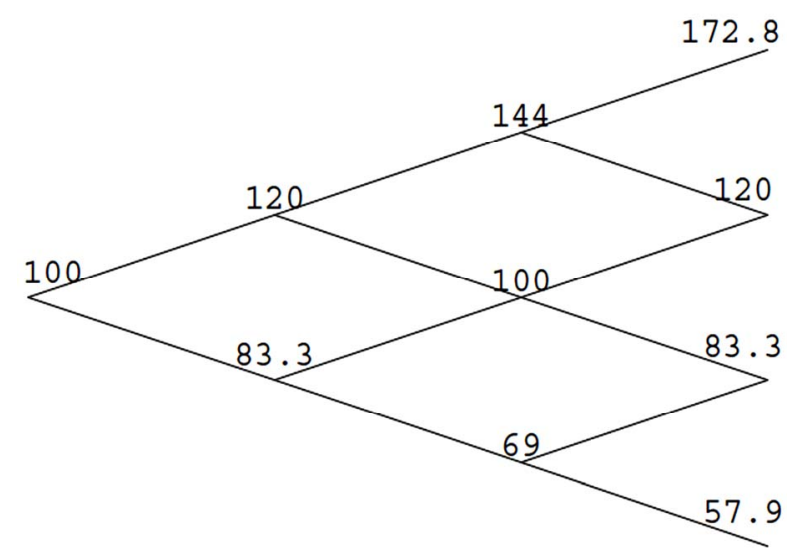

Figure 1. Market values in Leland's example.

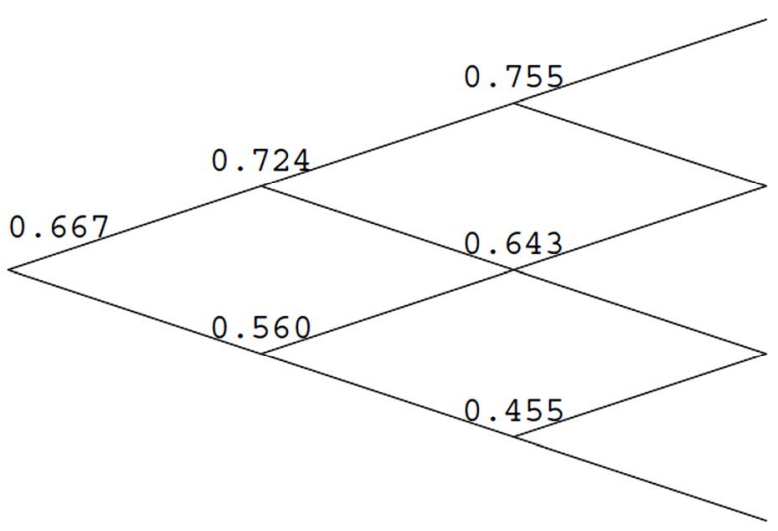

Figure 2. Implied Probabilities for call-option portfolio with $K=100$.
Leland concludes that this investor is mean-averting in the sense that an up-move always implies a larger (or equal) probability of a further up-move than the probability for an up-move in the step before. The analogous property holds for down moves.

A further example in [2] with $K=110$ gives a similar result, i.e. again mean aversion of the investor. Leland moreover gives some informal remarks on the $N$-step case ("there seems to be mean aversion at nodes with stock values near to the initial stock value $S_{0}$ ") and two examples for path-dependent contingent claims in a 3-step model (here he detected rather diffuse individual expectations).

We felt that a more general discussion is necessary to obtain valid conclusions. So in the following we will try to explicitely determine all contingent claims (i.e. dynamic trading strategies) which can be considered by a strictly mean-averting, respectively by a strictly mean-reverting investor in a binomial $N$-step model and in the BlackScholes model. Here we use the following definition of a strictly mean-averting investor (resp. mean-reverting investor) in a binomial model (the definition for the BlackScholes model will be given later).

Definition 2.1. We call an investor strictly mean-averting if his trading strategy induces individual expectations with the following properties

$$
p\left(\left(v_{0}, \cdots, v_{k-2}\right) S_{0} ; k-1\right) \leq p\left(\left(v_{0}, \cdots, v_{k-2}, u\right) S_{0} ; k\right)
$$

and

$$
1-p\left(\left(v_{0}, \cdots, v_{k-2}\right) S_{0} ; k-1\right) \leq 1-p\left(\left(v_{0}, \cdots, v_{k-2}, d\right) S_{0} ; k\right)
$$

for all $v_{0}, \cdots, v_{k-2} \in\{u, d\} ; k=1, \cdots, N-1$.

The investor will be called strictly mean-reverting if the "less or equal-sign" is replaced by the "larger or equal sign" in both inequalities. In the following we will call the corresponding strategies either mean-averting strategies resp. mean-reverting strategies.

For path-independent strategies the above properties reduce to

$$
\begin{gathered}
p\left(u^{i} d^{k-1-i} S_{0} ; k-1\right) \leq p\left(u^{i+1} d^{k-1-i} S_{0} ; k\right) \\
1-p\left(u^{i} d^{k-1-i} S_{0} ; k-1\right) \leq 1-p\left(u^{i} d^{k-i} S_{0} ; k\right)
\end{gathered}
$$

for $i=0, \cdots, k-1 ; k=1, \cdots, N-1$.

For our investigations we further need a suitable notion for contingent claims (i.e. for trading strategies) in our models. We denote trading strategies by

$$
W:=\left\{W\left(\left(v_{0}, \cdots, v_{N-1}\right)\right) ; v_{i} \in\{u, d\}\right\}
$$

where $W\left(\left(v_{0}, v_{1}, \cdots, v_{N-1}\right)\right)$ denotes the payoff of the strategy if path $\left(v_{0}, \cdots, v_{N-1}\right)$ happens. In the path-independent case this reduces to 


$$
W:=\left\{W\left(u^{i} d^{N-i} S_{0}\right) ; i=0, \cdots, N\right\} .
$$

We will also use the notation $W_{i}:=W\left(u^{i} d^{N-i} S_{0}\right)$. We restrict to "admissible" trading strategies, i.e. to strategies with $W\left(\left(v_{0}, v_{1}, \cdots, v_{N-1}\right)\right) \geq 0$ always.

In later sections we will proceed by induction, and it will turn out that much of the work is already contained in the full discussion of a 2-step model. This will be done in the next two sections.

\section{Mean-Averting Investors in the Two-Step Model: The Path-Independent Case}

We start with our "reverse engineering" in the 2-step model by assuming an optimal strategy (portfolio) $W$, the average utility function $U$ and by calculating from this the investor expectations $p$. An arbitrary strategy in the 2-step case is given by

$$
W=\{W(u, u), W(u, d), W(d, u), W(d, d)\} .
$$

The price at time zero of the strategy is

$$
P_{u}^{2} W(u, u)+P_{u} P_{d} W(u, d)+P_{d} P_{u} W(d, u)+P_{d}^{2} W(d, d) .
$$

Since we compare strategies $W$ with the average strategy of buying the market portfolio $S$ we have the budget constraint

$$
S_{0}=P_{u}^{2} W(u, u)+P_{u} P_{d} W(u, d)+P_{d} P_{u} W(d, u)+P_{d}^{2} W(d, d) .
$$

The trader following $W$ is maximizing his utility

$$
\begin{aligned}
& p\left(S_{0} ; 0\right) p\left(u S_{0} ; 1\right) U(W(u, u)) \\
& +p\left(S_{0} ; 0\right)\left(1-p\left(u S_{0} ; 1\right)\right) U(W(u, d)) \\
& +\left(1-p\left(S_{0} ; 0\right)\right) p\left(d S_{0} ; 1\right) U(W(d, u)) \\
& +\left(1-p\left(S_{0} ; 0\right)\right)\left(1-p\left(d S_{0} ; 1\right)\right) U(W(d, d)) .
\end{aligned}
$$

Hence by Lagrange we obtain the equations

$$
\begin{aligned}
& \lambda P_{u}^{2} \tilde{U}(W(u, u))=p\left(S_{0} ; 0\right) p\left(u S_{0} ; 1\right) \\
& \lambda P_{u} P_{d} \tilde{U}(W(u, d))=p\left(S_{0} ; 0\right)\left(1-p\left(u S_{0} ; 1\right)\right) \\
& \lambda P_{d} P_{u} \tilde{U}(W(d, u))=\left(1-p\left(S_{0} ; 0\right)\right) p\left(d S_{0} ; 1\right) \\
& \lambda P_{d}^{2} \tilde{U}(W(d, d))=\left(1-p\left(S_{0} ; 0\right)\right)\left(1-p\left(d S_{0} ; 1\right)\right)
\end{aligned}
$$

where $\tilde{U}=\frac{1}{U^{\prime}}$ (for our special case $\tilde{U}(W)=W^{\alpha}$ ). The sum of the right hand sides is 1 , so that

$$
\lambda=\frac{1}{E^{*}\left(\tilde{U}(W) \mid S_{0}\right)}
$$

where $E^{*}$ denotes expectation with respect to the risk neutral measure. This of course easily generalizes in obvious form to higher step number. Since in this section we are interested in traders whose optimal contingent claim turns out to be path-independent, we have

$$
W(u, d)=W(d, u)
$$

for simplicity we use the notation:

$$
W_{0}=W(d, d), W_{1}=W(d, u)=W(u, d), W_{2}=W(u, u)
$$

and $p_{0}=p\left(S_{0} ; 0\right), p_{1}=p\left(u S_{0} ; 1\right), p_{2}=p\left(d S_{0} ; 1\right)$.

It is easily checked that (1),(2),(3) has a unique solution $p_{0}, p_{1}, p_{2}$, namely

$$
\begin{aligned}
p_{2} & =\frac{P_{u} W_{1}^{\alpha}}{P_{u} W_{1}^{\alpha}+P_{d} W_{0}^{\alpha}}=\frac{P_{u} E^{*}\left(\tilde{U}(W) \mid S_{2}=u d S_{0}\right)}{E^{*}\left(\tilde{U}(W) \mid S_{1}=d S_{0}\right)} \\
p_{1} & =\frac{P_{u} W_{2}^{\alpha}}{P_{u} W_{2}^{\alpha}+P_{d} W_{1}^{\alpha}}=\frac{P_{u} E^{*}\left(\tilde{U}(W) \mid S_{2}=u^{2} S_{0}\right)}{E^{*}\left(\tilde{U}(W) \mid S_{1}=u S_{0}\right)} \\
p_{0} & =\frac{P_{u}\left(P_{u} W_{2}^{\alpha}+P_{d} W_{1}^{\alpha}\right)}{P_{u}\left(P_{u} W_{2}^{\alpha}+P_{d} W_{1}^{\alpha}\right)+P_{d}\left(P_{u} W_{1}^{\alpha}+P_{d} W_{0}^{\alpha}\right)} \\
& =\frac{P_{u} E^{*}\left(\tilde{U}(W) \mid S_{1}=u S_{0}\right)}{E^{*}\left(\tilde{U}(W) \mid S_{0}=S_{0}\right)}
\end{aligned}
$$

$\left(S_{i}\right.$ is the price of the market portfolio at time $i$ ).

In these relations $W_{0}$, via the budget constraint (1), is uniquely determined by $W_{1}$ and $W_{2}$. We only consider admissible strategies, i.e. $W_{0}, W_{1}, W_{2} \geq 0$. So the definition region for $W$ is the triangle in Figure 3.

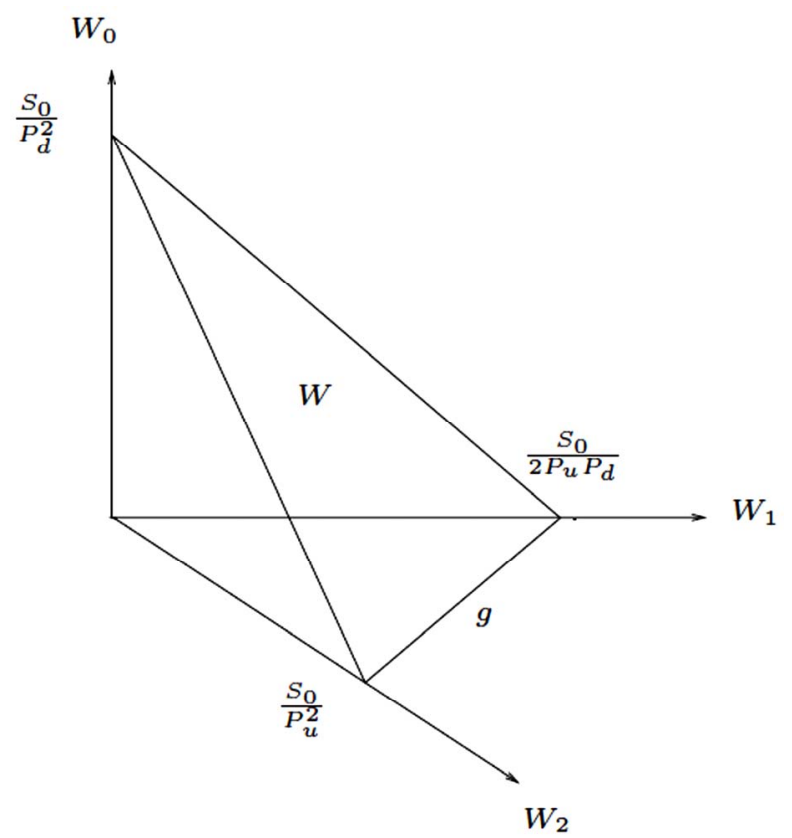

Figure 3. Definition region for strategy $W$. 
Strict mean-aversion now is determined by $p_{0} \leq p_{1}$ and $1-p_{0} \leq 1-p_{2} \Leftrightarrow p_{2} \leq p_{0}$.

Easy calculation based on (4) shows that the two conditions are equivalent and reduce to the single vivid condition

$$
W_{1}^{2} \leq W_{0} W_{2}
$$

(so in any case there are no investors who are neither meanaverting nor mean-reverting).

It is worth noting that the condition is in no way dependent on $\alpha$ or on the average expectations $\pi_{u}$ and $\pi_{d}$. It is dependent on $P_{u}$ only via the dependence of $W_{0}$ on $W_{1}$, $W_{2}$ through the budget constraint: inserting

$$
W_{0}=\frac{S_{0}}{P_{d}^{2}}-2 W_{1} \frac{P_{u}}{P_{d}}-W_{2}\left(\frac{P_{u}}{P_{d}}\right)^{2}
$$

into (5) leads to

$$
0 \leq W_{1} \leq \frac{-W_{2} P_{u}+\sqrt{S_{0} W_{2}}}{P_{d}}=\beta\left(W_{2}\right) .
$$

This determines the region for strictly mean-averting investors $A$ and for strictly mean-reverting investors $R$.

The boundary $\tilde{\beta}$ belongs to both regions. $g$ and the $W_{1}$-axis are tangents to $\beta$, that is the projection of $\tilde{\beta}$ onto the plane $W_{1} W_{2}$ (See Figures 4 and 5). Buying the market portfolio is mean-averting and mean-reverting, hence it is located on $\tilde{\beta}$. (Just insert $W_{2}=u^{2} S_{0}$, $W_{1}=S_{0}, W_{0}=\frac{S_{0}}{d^{2}}$ to check this once more).

$W_{1}$

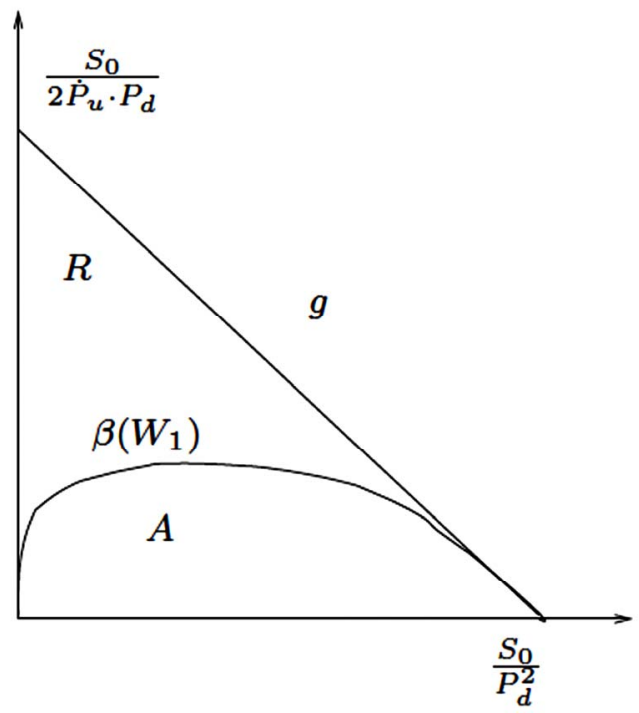

$W_{2}$

Figure 4. Projection of $\tilde{\beta}$ to the $W_{1}, W_{2}$-plane.
$W_{0}$

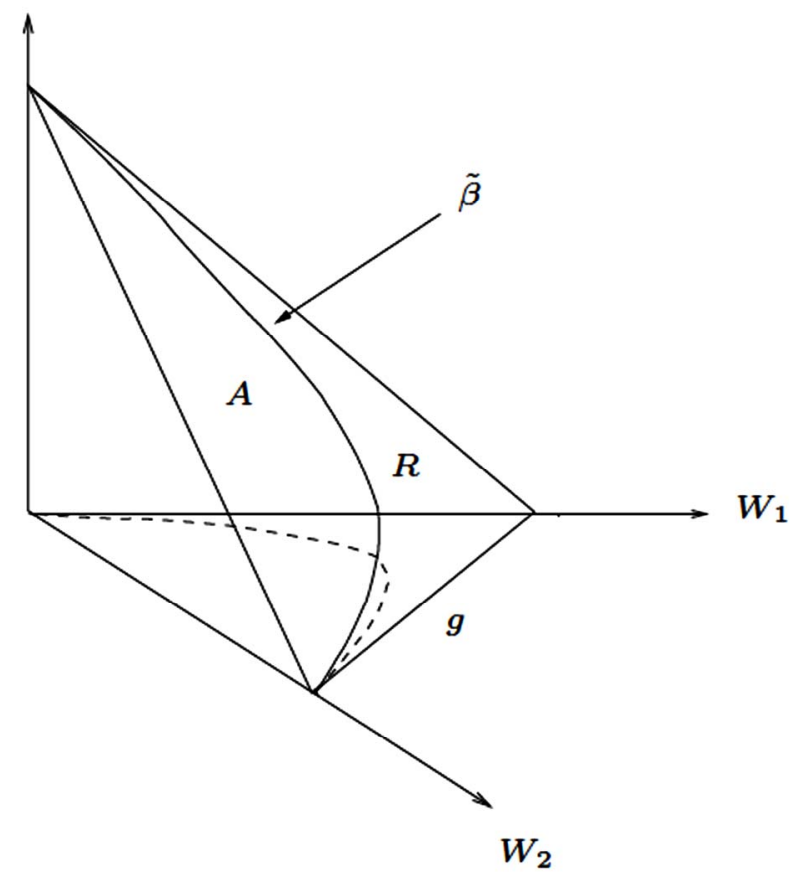

Figure 5. Curve $\tilde{\beta}$ separating mean-averting $(A)$ and mean-reverting $(R)$ strategies $W$.

\section{Mean-Averting Investors in the Two-Step Model: The Path-Dependent Case}

We just have to return to the system (2), now with $W(d, u)$ and $W(u, d)$ not necessarily being equal. We write $W_{1}:=W(d, u)$ and $\tilde{W}_{1}:=W(u, d)$, solve (2) and obtain now

$$
\begin{aligned}
p_{2} & =\frac{P_{u} W_{1}^{\alpha}}{P_{u} W_{1}^{\alpha}+P_{d} W_{0}^{\alpha}}=\frac{P_{u} E^{*}\left(\tilde{U}(W) \mid S_{2}=d u S_{0}\right)}{E^{*}\left(\tilde{U}(W) \mid S_{1}=d S_{0}\right)} \\
p_{1} & =\frac{P_{u} W_{2}^{\alpha}}{P_{u} W_{2}^{\alpha}+P_{d} \tilde{W}_{1}^{\alpha}}=\frac{P_{u} E^{*}\left(\tilde{U}(W) \mid S_{2}=u^{2} S_{0}\right)}{E^{*}\left(\tilde{U}(W) \mid S_{1}=u S_{0}\right)} \\
p_{0} & =\frac{P_{u}\left(P_{u} W_{2}^{\alpha}+P_{d} \tilde{W}_{1}^{\alpha}\right)}{P_{u}\left(P_{u} W_{2}^{\alpha}+P_{d} \tilde{W}_{1}^{\alpha}\right)+P_{d}\left(P_{u} W_{1}^{\alpha}+P_{d} W_{0}^{\alpha}\right)} \\
& =\frac{P_{u} E^{*}\left(\tilde{U}(W) \mid S_{1}=u S_{0}\right)}{E^{*}\left(\tilde{U}(W) \mid S_{0}=S_{0}\right)}
\end{aligned}
$$

The two mean-averting conditions

$$
p_{0} \leq p_{1} \quad \text { and } \quad 1-p_{0} \leq 1-p_{2}
$$

now are not equivalent in general. Inserting (6) into (7) leads to the following two mean-aversion conditions

$$
P_{d} \tilde{W}_{1}^{2 \alpha}+P_{u} \tilde{W}_{1}^{\alpha} W_{2}^{\alpha} \leq P_{u} W_{1}^{\alpha} W_{2}^{\alpha}+P_{d} W_{0}^{\alpha} W_{2}^{\alpha}
$$


and $P_{u} W_{1}^{2 \alpha}+P_{d} W_{1}^{\alpha} W_{0}^{\alpha} \leq P_{d} W_{0}^{\alpha} \tilde{W}_{1}^{\alpha}+P_{u} W_{0}^{\alpha} W_{2}^{\alpha}$

For $W_{1}=\tilde{W}_{1}$ (i.e. path-independence) (8) and (9) are equivalent, of course. If $W_{1} \leq \tilde{W}_{1}$ then we easily check that (8) implies (9). If $\tilde{W}_{1} \leq W_{1}$ then (9) implies (8).

Hence, an investor is strictly mean-averting if and only if $W_{1} \leq \tilde{W}_{1}$ and (8) holds or $W_{1} \geq \tilde{W}_{1}$ and (9) holds.

Let $(\tilde{8})$ and $(\tilde{9})$ denote Equations (8) and (9) with "greater-or-equal-sign", i.e. $(\tilde{8})$ and $(\tilde{9})$ are the conditions for strictly mean-reverting traders, then for $W_{1}=\tilde{W}_{1}(\tilde{8})$ and $(\tilde{9})$ are equivalent. If $W_{1} \leq \tilde{W}_{1}$ then ( $\tilde{9})$ implies $(\tilde{8})$ and if $W_{1} \geq \tilde{W}_{1}$ then $(\tilde{8})$ implies $(\tilde{9})$.

Hence, an investor is strictly mean-reverting if and only if $W_{1} \leq \tilde{W}_{1}$ and (9) does not hold or $W_{1} \geq \tilde{W}_{1}$ and (8) does not hold.

Finally, an investor is neither mean-averting nor meanreverting if and only if $W_{1}<\tilde{W}_{1}$ and (9) holds but (8) does not hold or $W_{1}>\tilde{W}_{1}$ and (8) holds but (9) does not hold.

The conditions now (in the path-dependent case) depend on the model and (via $\alpha$ ) on the expectations of the average investor. To obtain concrete explicit mean-averting strategies we still have to insert for $W_{0}$ from the budget constraint. The subsequent example should serve as an illustration. Following Leland we choose the parameters

$$
u=1.2 ; \quad \pi_{u}=\frac{2}{3}, \text { hence } \alpha=2.4
$$

and we set $\tilde{W}_{1}=K W_{1}$ with $K=1$ (path-independent case, Figure 6), $K=0.8$ (Figure 7) and $K=1.2$ (Figure 8). We just show the $W_{1}, W_{2}$-plane ( $W_{3}$ again then is uniquely determined by $W_{1}, W_{2}$ and $K$ ).

\section{Individual Investor Probabilities in a Binomial N-Step Model}

We now consider the $N$-step binomial model. First (like

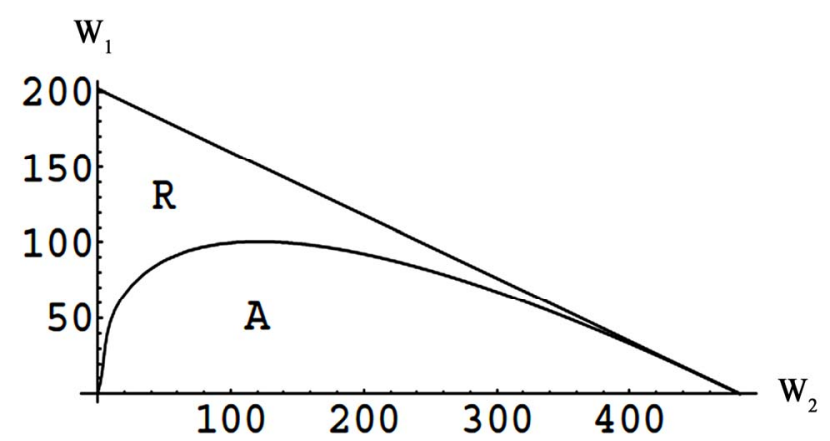

Figure 6. Curve separating mean-averting $(A)$ strategies and mean-reverting $(R)$ strategies for $K=1$ (projection to $W_{1}, W_{2}$-plane).

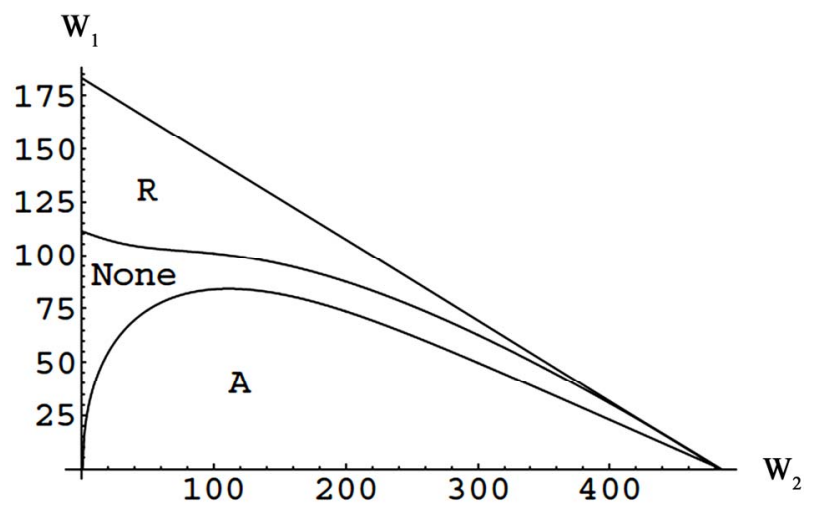

Figure 7. Curves separating mean-averting $(A)$ strategies, mean-reverting $(R)$ strategies and other strategies (None) for $K=0.8$ (projection to $W_{1}, W_{2}$-plane).

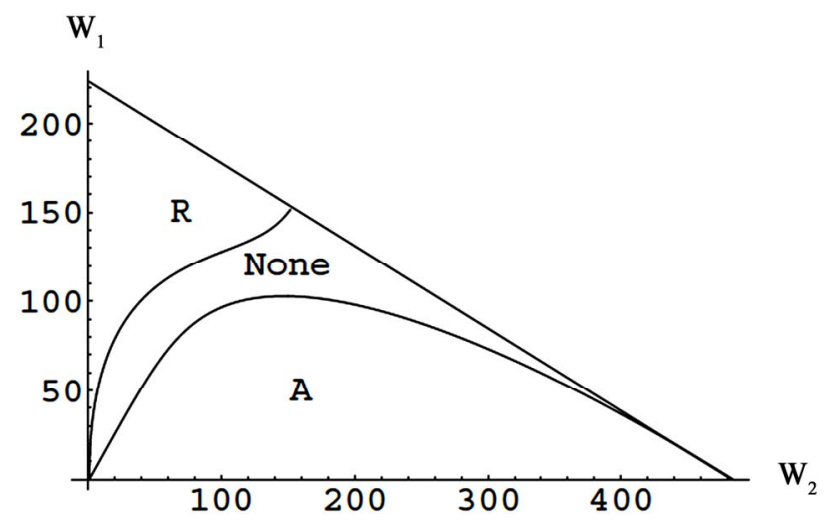

Figure 8. Curves separating mean-averting $(A)$ strategies, mean-reverting $(R)$ strategies and other strategies (None) for $K=1.2$ (projection to $W_{1}, W_{2}$-plane).

in Section 3) we again assume that a path-independent contingent claim is the optimal choice for the investor. We will give an explicit formula for the individual probabilities of an investor. As it is suggested from the results in the two-step case we will show the following

Theorem 5.1. For a given path-independent strategy $W$ the individual up-move probabilities $p\left(S_{i} ; i\right)$ are uniquely determined by

$$
p\left(S_{i} ; i\right)=\frac{P_{u} E^{*}\left(\tilde{U}(W) \mid S_{i+1}=u S_{i}\right)}{E^{*}\left(\tilde{U}(W) \mid S_{i}\right)}
$$

where $\tilde{U}=\frac{1}{U^{\prime}}$ (Note that this relation in fact holds for any utility function $U$ and not just for $\left.U(x)=\frac{x^{1-\alpha}}{1-\alpha}\right)$.

Proof. We use induction on $N$. For $N=2$ we know that the result holds. Since we assume that the results hold for $k$-step models with $k \leq N-1$, the formula (10) holds for all $p\left(S_{i} ; i\right)$ with $i \geq 1$ (see Figure 9).

So it remains to prove the formula for $p\left(S_{0} ; 0\right)$. To 
this end we use the first of the Lagrange Equations (2) in its $N$-step version

$\lambda P_{u}^{N} \tilde{U}(W(u, \cdots, u))=p\left(S_{0} ; 0\right) p\left(u S_{0} ; 1\right) \cdots p\left(u^{N-1} S_{0} ; N-1\right)$

with

$$
\lambda=\frac{1}{E^{*}\left(\tilde{U}(W) \mid S_{0}\right)} .
$$

Inserting for $p\left(u S_{0} ; 1\right), \cdots, p\left(u^{N-1} S_{0} ; N-1\right)$ gives

$$
\begin{aligned}
\frac{P_{u}^{N} \tilde{U}(W(u, \cdots, u))}{E^{*}\left(U(W) \mid S_{0}\right)}= & p\left(S_{0} ; 0\right) \frac{P_{u} E^{*}\left(\tilde{U}(W) \mid S_{2}=u^{2} S_{0}\right)}{E^{*}\left(\tilde{U}(W) \mid S_{1}=u S_{0}\right)} \\
& \cdots \frac{P_{u} E^{*}\left(\tilde{U}(W) \mid S_{N}=u^{N} S_{0}\right)}{E^{*}\left(\tilde{U}(W) \mid S_{N-1}=u^{N-1} S_{0}\right)}
\end{aligned}
$$

Since $E^{*}\left(\tilde{U}(W) \mid S_{N}=u^{N} S_{0}\right)=\tilde{U}(W(u, \cdots, u)$, the result also follows for $p\left(S_{0} ; 0\right)$.

We have stated and shown the result for the path-independent case first, because it is more intuitive. Following the proof we see that we can prove the path-dependent result in the same way (with the obvious notational adaptations). (Note that a path going through node $N_{1}\left(N_{2}\right)$ (see Figure 9) remains in $R_{1}\left(R_{2}\right)$ so we again can use the induction assumption).

Theorem 5.2. For a given path-dependent strategy $W$ the individual up- move probabilities in the N-step case $p\left(\left(v_{0} v_{1} \ldots v_{k-2}\right) S_{0} ; k-1\right)$ are uniquely determined by

$$
\begin{gathered}
p\left(\left(v_{0}, v_{1}, \cdots, v_{k-2}\right) S_{0} ; k-1\right) \\
=\frac{P_{u} E^{*}\left(\tilde{U}(W) \mid S_{k}=v_{0} v_{1} \cdots v_{k-2} u S_{0}\right)}{E^{*}\left(\tilde{U}(W) \mid S_{k-1}=v_{0} v_{1} \cdots v_{k-2} S_{0}\right)}
\end{gathered}
$$

where $v_{0}, v_{1}, \cdots, v_{k-1} \in\{u, d\}$ and $k=1, \cdots, N$. (This again holds for an arbitrary utility function $U$.)
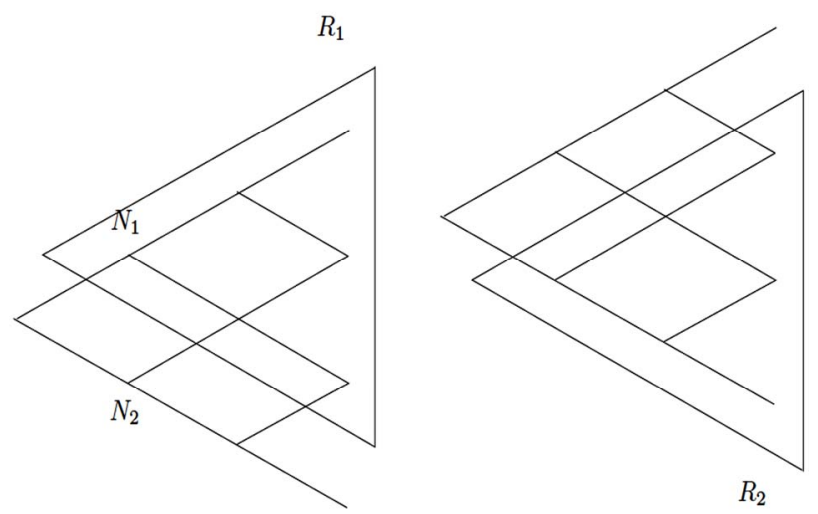

Figure 9. Regions $R_{1}, R_{2}$ where (10) holds by induction hypothesis.

\section{The Martingale Property of the Individual Expected Return of the Market Portfolio}

Leland notes that the process of returns of the market portfolio under the individual expectations is a martingale with respect to the filtration of the binomial model, i.e. for

$$
X_{i}:=\frac{S_{i+1}-S_{i}}{S_{i}} ; \quad i=0, \cdots, N-1
$$

we have $E\left(X_{j} \mid S_{i}\right)=E\left(X_{i} \mid S_{i}\right)$ for $i<j$. ( $E$ in this section denotes expectation with respect to the individual probabilities.) This fact easily can be obtained for general path-independent strategies from Theorem 5.1, respectively from looking at the Lagrangean system (2) (in general form).

The assertion (as also noted by Leland) is not at all true for path-dependent strategies, as for example is immediately seen from the geometric Asian future in Section 10.

Theorem 6.1. In the path-independent case we have

$$
E\left(X_{j} \mid S_{i}\right)=E\left(X_{i} \mid S_{i}\right) \quad \text { for } \quad i<j
$$

Proof. Since the market portfolio has constant up-anddown-move factors $u, d$ it suffices to show that

$$
\operatorname{Prob}\left(\text { up - move in step } j+1 \mid S_{i}\right)=p\left(S_{i}, i\right)
$$

(with respect to individual expectations). We show this property for $j=N-1$ and $i=0$. The method easily extends to general $i$ and $j$. We have

$$
\begin{aligned}
= & \sum_{v_{0}, \cdots, v_{N-2} \in\{u, d\}} q\left(v_{0}\right) \\
& \cdots q\left(v_{0}, \cdots, v_{N-3}\right) p\left(\left(v_{0}, \cdots, v_{N-2}\right) S_{0} ; N-1\right)
\end{aligned}
$$

where

$$
q\left(v_{0}, \cdots, v_{k}\right)=\left\{\begin{array}{ccc}
p\left(\left(v_{0}, \cdots, v_{k-1}\right) S_{0} ; k\right) & \text { if } & v_{k}=u \\
1-p\left(\left(v_{0}, \cdots, v_{k-1}\right) S_{0} ; k\right) & \text { if } & v_{k}=d
\end{array}\right.
$$

Using the Lagrangean Equations (2) the last sum equals

$$
\begin{aligned}
& \lambda \sum_{v_{0}, \cdots, v_{N-2} \in\{u, d\}} P_{v_{0}} \cdots P_{v_{N-2}} P_{u} W^{\alpha}\left(v_{0}, \cdots, v_{N-2}, u\right) \\
\stackrel{(*)}{=} & \lambda P_{u} E^{*}\left(\tilde{U}(W) \mid S_{1}=u S_{0}\right) \\
= & \frac{P_{u} E^{*}\left(\tilde{U}(W) \mid S_{1}=u S_{0}\right)}{E^{*}\left(\tilde{U}(W) \mid S_{0}\right)}=p_{0} .
\end{aligned}
$$

Note that the equality denoted by $\stackrel{(*)}{=}$ does not hold in general for path-dependent strategies. 


\section{Mean Averting Investors in a Binomial N-Step Model: The Path Independent Case}

Now we can explicitly classify the strictly mean-averting and mean-reverting strategies in the path-independent case.

Theorem 7.1. The path-independent strategy

$W=\left(W_{i}\right)_{i=0, \cdots, N}\left(W_{i}\right.$ is the strategy value at $\left.u^{i} d^{N-i} S_{0}\right)$ is strictly mean-averting if and only if

$$
W_{i}^{2} \leq W_{i-1} W_{i+1}
$$

for all $i=1, \cdots, N-1$.

$W_{i}$ is strictly mean-reverting if and only if

$$
W_{i}^{2} \geq W_{i-1} W_{i+1}
$$

for all $i=1, \cdots, N-1$.

\section{Remarks}

1) So we have constant individual probabilities if and only if $W_{i}=\left(W_{i-1} W_{i+1}\right)^{1 / 2}$ for all $i=1, \cdots, N-1$.

2) Trivially, for our special utility function $U$ inequality (11) is equivalent to $\tilde{U}_{i}^{2} \leq \tilde{U}_{i-1} \tilde{U}_{i+1}$ and (12) is equivalent to $\tilde{U}_{i}^{2} \geq \tilde{U}_{i-1} \tilde{U}_{i+1}$. We will refer to this later.

$\left(\tilde{U}_{k}:=\tilde{U}\left(W_{k}\right)\right)$

We conclude from Theorem 7.1 (taking the logarithm of both sides of (11) and of (12)) the following

Corollary 7.2. If $W_{i}>0$ for all $i$, then the path- independent strategy $W=\left(W_{i}\right)_{i=0, \cdots, N}$ is strictly meanaverting if and only if $\left\{\log W_{0}, \cdots, \log W_{N}\right\}$ is convex and the path-independent strategy $W=\left(W_{i}\right)_{i=0, \cdots, N}$ is strictly mean-reverting if and only if $\left\{\log W_{0}, \cdots, \log W_{N}\right\}$ is concave.

\section{Remarks}

3) If $W_{i} \geq 0$ for all $i$ and $W_{k}=0$ for some $\mathrm{k}$, then we have mean-aversion if and only if $\forall i, W_{i}=0$ except for $W_{0}$ and $W_{N}$ that can be $>0$.

4) If $W_{i} \geq 0$ for all $i$ and $W_{k}=0$ for some $k$, then we have mean-reversion if and only if $W_{k-1}=0$ or $W_{k+1}=0$ and always when there are at least three successive $W_{j}, W_{j+1}, W_{j+2}>0,\left\{\log W_{j}, \log W_{j+1}, \log W_{j+2}\right\}$ is concave.

Proof of the Theorem: 7.1. Again we use induction on $N$, and again we know that the assertion is true for $N=2$ and assume that it is true for $K \leq N-1$. Since we have mean-aversion in regions $R_{1}$ and $R_{2}$ of Figure 9 if and only if (11) holds for $W_{1}, \cdots, W_{N}$ resp. for $W_{0}, \cdots, W_{N-1}$ we see that $(11)$ is a necessary condition. Let now (11) be satisfied. Further let for all $j=0, \cdots, N-3$ the following condition hold true

$$
\left(P_{u} W_{j+3}^{\alpha}+P_{d} W_{j+2}^{\alpha}\right)\left(P_{u} W_{j+1}^{\alpha}+P_{d} W_{j}^{\alpha}\right) \geq\left(P_{u} W_{j+2}^{\alpha}+P_{d} W_{j+1}^{\alpha}\right)^{2}
$$

i.e. the condition (11) not only holds for the terminal wealth $W$ at the time $N$ but also for time $N-1$ for the values $E^{*}\left(\tilde{U}(W) \mid S_{N-1}\right)$ (see Figure 10).

Then by Theorem 5.1 and by induction hypothesis we have mean-aversion also for $R_{3}$ and hence for the whole strategy. We show that (11) implies (13) and the proof is finished. But this is just simple calculation (note that (11) $\Rightarrow W_{j+2}^{2} \leq W_{j+1} W_{j+3}$ and $\left.W_{j+1}^{2} \leq W_{j} W_{j+2} \Rightarrow W_{j+1} W_{j+2} \leq W_{j} W_{j+3}\right)$. Strictly mean-reverting strategies are treated quite analogously.

It seems to us to be much harder to explicitely classify the path-dependent strict mean-averting or reverting strategies.

Let us consider now an investor (again in the path-independent case) who is neither mean-averting nor meanreverting and let us ask the question at which nodes of the corresponding model we have local mean-aversion or reversion.

By the above investigations (especially on the 2-step case) it is clear how to detect all strings of local monotonicity of expectations: just fix a certain point in time $i$. Then calculate $E\left(\tilde{U}(W) \mid S_{i}\right)$ for all possible values of $S_{i}$. The resulting values (ordered from below) say $\bar{U}_{0}, \cdots, \bar{U}_{i}$ determine in which nodes $N_{0}, \cdots, N_{i-1}$ (ordered from below) of step $i-1$ we have local mean-aversion, or local mean-reversion. We have mean-aversion (reversion) in

$N_{0}$, iff $\bar{U}_{0}, \bar{U}_{1}, \bar{U}_{2}$ satisfy the mean-aversion (reversion) property in analogy to remark 2 ) in this section.

$N_{i-1}$, iff $\bar{U}_{i-2}, \bar{U}_{i-1}, \bar{U}_{i}$ satisfy the mean-aversion (reversion) property in analogy to remark 2 ) in this section

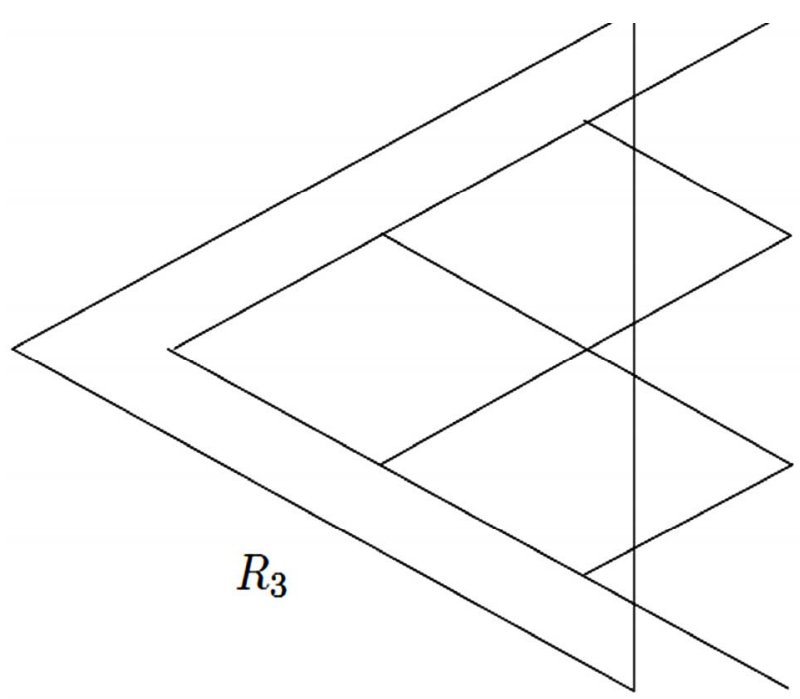

Figure 10. Region $R_{3}$ where (11) holds by induction hypothesis. 
$N_{j}, 0<j<i-1$, iff $\bar{U}_{j-1}, \bar{U}_{j}, \bar{U}_{j+1}, \bar{U}_{j+2}$ satisfy the meanaversion (reversion) property in analogy to remark 2 ) in this section.

We will illustrate this fact with an example in Section 9 (Figures 16 and 17).

\section{Mean-Averting and Mean-Reverting Investors in the Black-Scholes Model}

Until now we have restricted ourselves to the binomial $N$-step model. In this section we show how to extend the results for the path-independent case of earlier sections to the one-dimensional Black-Scholes model: here first we need a suitable definition of a strictly mean-averting (resp. mean-reverting) investor. Let $\chi$ be a simple contingent claim with payoff-function $\Phi=\Phi\left(S_{T}\right)$. Here we assume that $\Phi$ is left (or right) continuous and $\Phi>0$. Since geometric Brownian motion

$$
\mathrm{d} S_{t}=\mu S_{t} \mathrm{~d} t+\sigma S_{t} \mathrm{~d} B_{t} \quad t \in[0, T]
$$

can be approximated by a binomial model with parameters

$$
\begin{gathered}
N=\frac{T}{\mathrm{~d} t} \\
u(N)=e^{\sigma \sqrt{\mathrm{d} t}} \\
\pi_{u}=\frac{1}{2}+\frac{\mu \sqrt{\mathrm{d} t}}{2 \sigma}
\end{gathered}
$$

(again we set $r=0$ ), implying for the utility exponent

$$
\alpha(N)=\frac{1}{2}+\frac{\log (\sigma+\mu \sqrt{\mathrm{d} t}-\log (\sigma-\mu \sqrt{\mathrm{d} t}))}{2 \sigma \sqrt{\mathrm{d} t}},
$$

a given strategy $W(N):=\left\{W_{i}(N)\right\}_{i=0, \cdots, N}$ should approximate $\Phi\left(S_{T}\right)$, i.e. $W_{i}(N)=\Phi\left(u(N)^{2 i-N} S_{0}\right)$. It is tempting to define, that the investor is strictly mean-averting in the Black-Scholes model, if he is strictly mean-averting in all these binomial models with $N \geq N_{0}$ large enough. This, by Theorem 7.1, is given if and only if for all $N$ large enough $\left(\log \Phi\left(u(N)^{2 i-N} S_{0}\right)\right)_{i=0, \cdots, N}$ is convex. Since $u(N) \rightarrow 1$ for $N$ to infinity, and since $\Phi$ is one-sided continuous, this is satisfied if and only if the function $g(x):=\log \left(\Phi\left(e^{x}\right)\right)$ is convex on $\mathbb{R}$.

Conversely, a strategy, i.e. a contingent claim is strictly mean-reverting if and only if $g(x)$ is concave. Note, that again the parameters $\mu$ and $\sigma$ do not influence the mean-aversion property.

\section{Example for the Path-Independent Case: The Call Option}

Of course (from the definition, respectively from the model setting) investing in the market portfolio is as well a mean-averting as a mean-reverting strategy. Obviously as well in the binomial model as in the Black-Scholes model our conditions are satisfied:

$$
\begin{aligned}
& \text { Binomial N-step } \\
& \qquad W_{i}=u^{2 i-N} S_{0} \\
& \log W_{i}=(2 i-N) \log u+\log S_{0} \quad \text { linear in } i .
\end{aligned}
$$

Black-Scholes

$$
\begin{array}{r}
\Phi(x)=x \\
g(x)=\log \left(\Phi\left(e^{x}\right)\right)=x \quad \text { linear in } x .
\end{array}
$$

Let us consider now buying call-options. To avoid uninteresting discussions of different cases we consider portfolios of a call-option together with a positive (at least minimal) amount of cash $c$, i.e., a strictly positive trading strategy.

The binomial $N$-step model:

$$
W_{i}=c+\operatorname{Max}\left(u^{2 i-N} S_{0}>K, 0\right)
$$

The values $\log W_{i}$ lie on a curve of the form in the Figure 11.

So in any case we have neither convexity nor concavity if there are at least three values for $i$ with $u^{2 i-N} S_{0}>K$. If there are two values for $i$ with $u^{2 i-N} S_{0}>K$ then accidentally we can have convexity. This indeed was the case in the two examples used by Leland with $N=3$ (see Figures 12 and 13).

If we however choose exactly Leland's parameters but $K=70$ then (see Figure 14) we do not have convexity any more.

Indeed the distribution of implied expectations in this example is given as in Figure 15.

\section{i Log $W$}

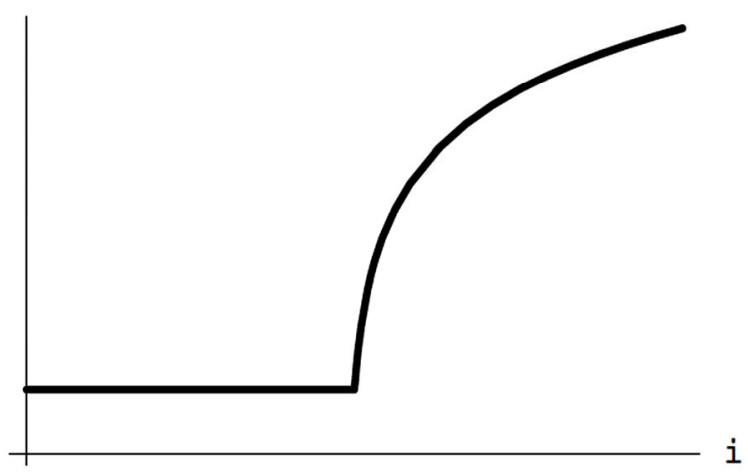

Figure 11. Logarithmic payoff of a call option portfolio. 


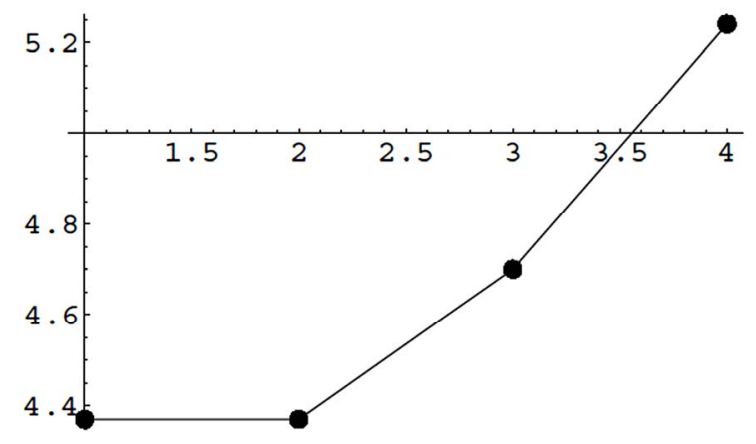

Figure 12. Logarithmic convex payoff, Leland: Example 1, $K=100$.

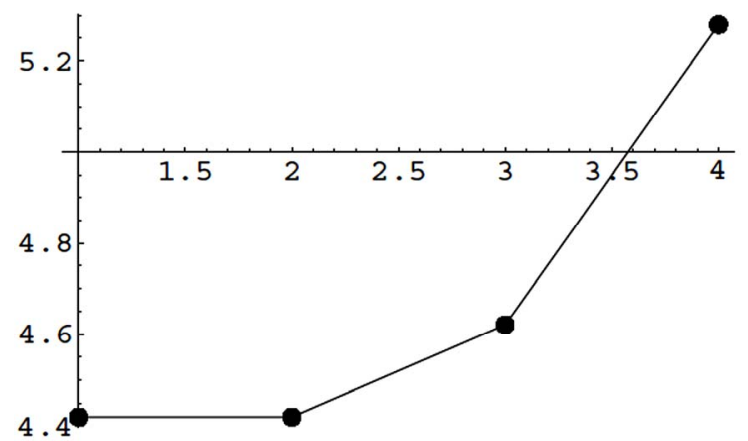

Figure 13. Logarithmic convex payoff, Leland: Example 2, $K=110$.

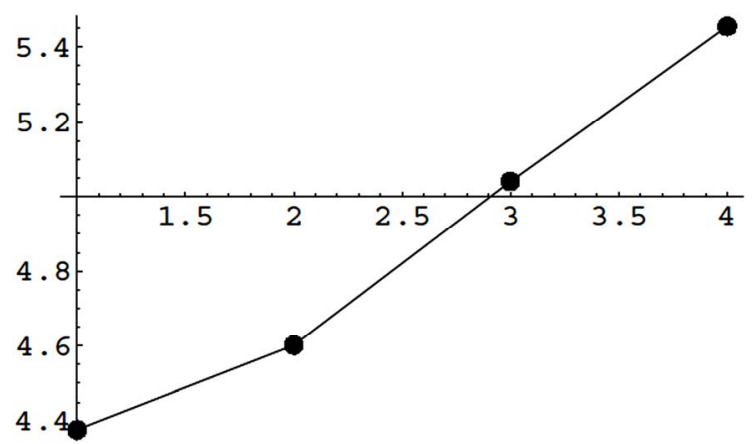

Figure 14. Logarithmic non-convex payoff Leland's example with $K=70$.

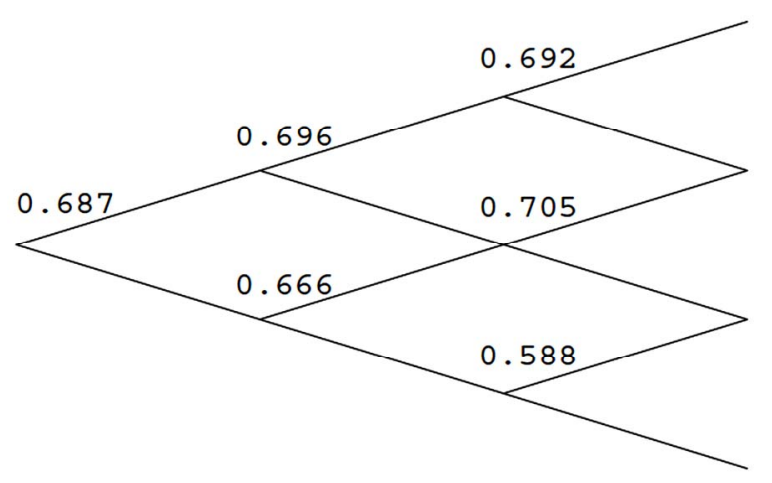

Figure 15. Implied expectations Leland's example with $K=70$.
Leland states that for larger $N$ we recognize meanaversion (when buying a call option) for nodes near to the initial wealth of the market portfolio. Our above discussion (and the discussion at the end of Section 7) shows that this is the case, but not necessarily near to the initial wealth but rather near to the strike $K$ and for nodes which lead only to end nodes out of the money (see the example in Figure 16 with the parameters of Leland's example 1 and with $N=10$. Here you find the up-probability in each node. Figure 17 shows the same situation as in Figure $\mathbf{1 6}$ with a square in each node that is not mean-averting).

\section{A Path-Dependent Example: Geometric Asian Future}

In [2] Leland considers arithmetic Asian futures in a 3-step model. To illustrate the result of Theorem 5.2 we have calculated the implied probabilities for a geometric Asian future. For a path $v_{0}, \cdots, v_{N-1}$ the payoff of this future is given by the geometric mean of the path-values:

$$
\left(S_{0}^{N+1} v_{0}^{N} v_{1}^{N-1} \cdots v_{N-1}\right)^{1 / N+1}
$$

(we omit the substraction of $S_{0}$ as it would be usual).

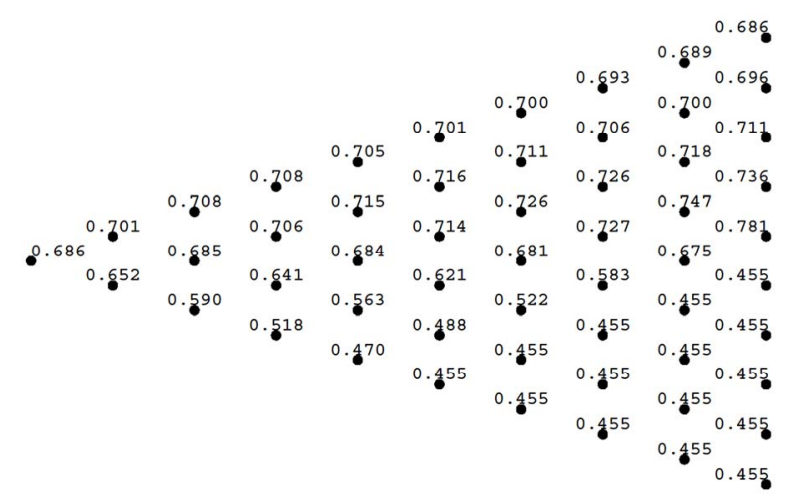

Figure 16. Implied probabilities, Leland's Example 1 with $N=10$.

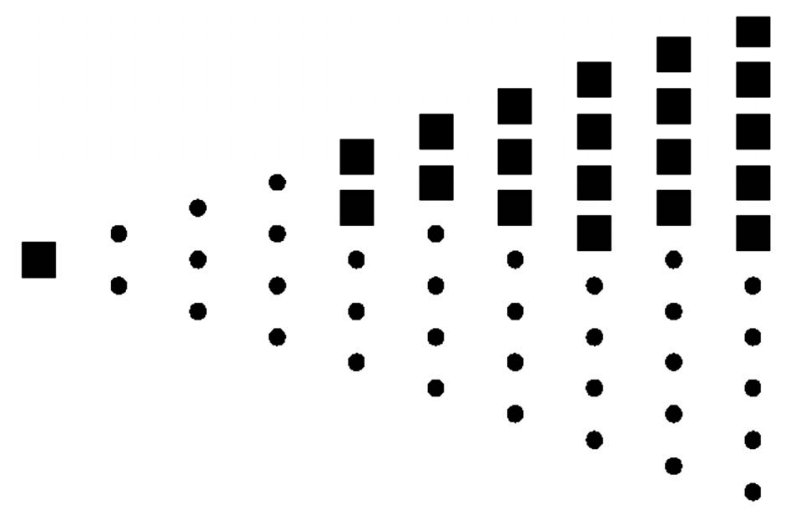

Figure 17. Mean-averting (•) and non-mean-averting (घ) nodes in Leland's Example 1 with $\mathbf{N}=10$. 
By Theorem 5.2 we now calculate the individual expectations

$$
\begin{aligned}
& p\left(\left(v_{0}, \cdots, v_{k-1}\right) S_{0} ; k\right)=\frac{P_{u} E^{*}\left(\tilde{U}(W) \mid S_{k}=v_{0} v_{1} \ldots v_{k-2} u S_{0}\right)}{E^{*}\left(\tilde{U}(W) \mid S_{k-1}=v_{0} v_{1} \ldots v_{k-2} S_{0}\right)} \\
= & \frac{P_{u} u^{\alpha \frac{N-k}{N+1}} E^{*}\left(w_{1}^{N-k} w_{2}^{N-K-1} \cdots w_{N-k}\right)}{P_{u} u^{\alpha \frac{N-k}{N+1}} E^{*}\left(w_{1}^{N-k} w_{2}^{N-k-1} \cdots w_{N-k}\right)+P_{d} d^{\alpha \frac{N-k}{N+1}} E^{*}\left(w_{1}^{N-k} w_{2}^{N-k-1} \cdots w_{N-k}\right)}=\frac{P_{u} u^{\alpha \frac{N-k}{N+1}}}{P_{u} u^{\alpha \frac{N-k}{N+1}}+P_{d} d^{\alpha \frac{N-k}{N+1}}}
\end{aligned}
$$

where $w_{i} \in\{u, d\}$.

Hence the individual probabilities in this example are independent of the path, and they even are independent of the value of the market portfolio, so we can write $p(k)$ shorthand. For $N$ large we have

$$
p(0) \sim \frac{P_{u} u^{\alpha}}{P_{u} u^{\alpha}+P_{d} d^{\alpha}}=\pi_{u}
$$

by the definition of $\alpha$ and

$$
p(N-1) \sim \frac{P_{u}}{P_{u}+P_{d}}=P_{u} .
$$

In any case we do not have mean-aversion or meanreversion.

It is also easily checked (by using the definition of $\alpha$ in Section 2) that $p(k)$ is monotonically increasing iff $P_{u} \geq \pi_{u}$ and monotonically decreasing iff $P_{u} \leq \pi_{u}$.

\section{Conclusions}

Based on the article [2] of Leland we have developed some techniques to calculate and to interpret the individual probabilities of an investor with average risk aversion but following non-average investing strategies. It is possible with the help of these techniques to completely discuss path-independent strategies. Thereby we could partly confirm assertions of Leland, partly we had to slightly adapt these assertions. For example we can conclude that in general the strategy obtained by combining a call option and cash is not a mean-averting strategy during the entire time to maturity.

Based on the developed techniques a lot of further future work should be possible: for example a classification of mean-averting path-dependent strategies, the extension to arbitrary discrete market models or to American options and the inclusion of transaction costs would be a most interesting topic.

\section{Acknowledgements}

We thank Gunther Leobacher and Friedrich Pillichshammer for technical support.

\section{References}

[1] H. E. Leland, "Who Should Buy Portfolio Insurance?" Journal of Finance, Vol. 35, No. 2, 1980, pp. 581-594. doi: $10.2307 / 2327419$

[2] H. E. Leland, "Options and Expectations," Institute of Business and Economic Research, University of California, Berkeley, 1996, pp. 43-51.

[3] M. Brennan, "The pricing of Contingent Claims in Discrete Time Models," Journal of Finance, Vol. 34, No. 1, 1979, pp. 53-68. doi:10.2307/2327143 